\title{
Spatial Processing for Frequency Diversity Schemes
}

\author{
Miguel Angel Lagunas, Fellow, IEEE, Ana I. Pérez Neira, Member, IEEE, Moeness G. Amin, Senior Member, IEEE, \\ and Josep Vidal, Associate Member, IEEE
}

\begin{abstract}
A novel technique to obtain optimum blind spatial processing for frequency diversity spread spectrum (FDSS) communication systems is introduced. The sufficient statistics for a linear combiner, which prove ineffective due to the interferers frequency characteristics, are modified to yield improved detection under partial jamming in the spectral domain. Robustness to partial time jamming is achieved by extending the notion of replicas over the frequency axis to a repetition over the time variable. Analysis and simulations are provided, showing the advantages of using FDSS with spatial diversity to combat the interference when it is confined to a narrow frequency band or short time interval relative to the desired signal extent in either domain.
\end{abstract}

\section{INTRODUCTION}

$\mathbf{F}$ REQUENCY diversity spread spectrum (FDSS) has been recently shown [1], [2] to be a powerful tool for digital detection as well as an effective alternative to the traditional spread spectrum techniques, namely, direct sequence (DSSS) and frequency hopping (FHSS) [3]. In a general context, diversity is conceived by the existence of several replicas (either in code, time, space, or frequency). When diversity is available to the receiver either by the structure of the transmitted signal or the architecture of the receiver, optimum spatial signal processing, which is blind to the temporal signal characteristics, can be derived.

In this paper, we consider the spatial-frequency diversity problem in wireless communication systems. We derive optimum spatial processing for FDSS systems under partial band jamming. The principle condition for the optimality in these systems is to have at least one frequency band contaminated by the interference and another band that is jammer free. These two bands will here in be referred to as the "hit" and "free" bands, respectively.

In partial band jamming, the above condition is often satisfied, as the desired signal is present in all the frequency bands in which the transmission bandwidth is slotted, whereas the jammer is only active in few bands due to its narrowband frequency characteristics. In this case, the optimum spatial combiners can be obtained in a two-step design procedure as follows. First, the spatial combiner of the free band is computed

Manuscript received September 4, 1998; revised June 21, 1999. This work was supported by CICYT (TIC96-0500-c10-01,TIC98-0412, TIC98-0703) of Spain and CIRIT (1998GR-00081) of Catalunya. M. G. Amin was supported in part by the Office of Naval Research under Grant N00014-98-1-0176. The associate editor coordinating the review of this paper and approving it for publication was Prof. José R. Casar.

M. A. Lagunas, A. I. Pérez Neira, and J. Vidal are with the Signal Theory and Communications Department, Universitat Politecnica de Catalunya, Barcelona, Spain (e-mail: miguel@gps.tsc.upc.es).

M. G. Amin is with the Department of Electrical and Computer Engineering, Villanova University, Villanova, PA 19085 USA.

Publisher Item Identifier S 1053-587X(00)00978-8. by minimizing the mean square error (MSE) between the spatial combiners' outputs of the free and the hit bands. In order to avoid trivial solutions, leading to a null beamformer or simultaneous removal of the desired signal in all bands, the cross correlation of the two band outputs is set equal to a nonzero value. Once the spatial combiner for the free band is provided, the corresponding output signal is used as a time reference to design the spatial combiners for the rest of the bands. It is shown that this spatial design procedure is blind to the desired signal waveform and does not require the proper labeling to identify the hit and the free bands to reach the optimum solution.

The sum of the likelihood of the different bands forms the sufficient statistics for optimal symbol by symbol detection. This requires the knowledge of the jammer level in every hit band [1]. On the other hand, the sufficient statistics in a suboptimum receiver is formed only from the bands free of jammer energy. The performances of both receivers, however, become approximately the same under high jammer-to-noise ratio in the hit bands. The offering of spatial processing to improved detection is in its ability to remove the jammer contamination in the hit bands prior to incorporating them into the detection scheme. The quality of jammer suppression in the hit bands, however, depends on the angular separation of the waveforms received by the different antennas. As the jammer and desired signal become closely spaced, removal of the jammer power through linear combining of signal arrivals renders them ineffective. In this case, suboptimal and optimum receivers converge to the same solution. It is noted that shadowing occurs when the angles of arrival of the jammer and the desired signal coincide. In this paper, we present the proper mechanism to form a sufficient statistic robust to the shadowing effect.

Section II provides the general structure of the FDSS systems as well as the mathematical description of the desired signal. Section III establishes the optimum receiver for the frequency diversity spread spectrum signal and presents the decision variable and probability of error under partial band jamming. In Section IV, we introduce spatial processing for improved detection in FDSS systems and derive the optimum solution for the spatial weights in the hit and free bands. Section V describes the optimum receiver that is robust to the shadowing effect. Section VI includes an adaptive algorithm as an alternative to the block processing described in Section IV. Section VII reports key features of the proposed design procedure for optimum spatial processing. It is shown that any information transmission system using coded waveforms, such as FDSS, permits blind optimum spatial processing, implying that the allocation of dedicated time or frequency slots for reference framing is unnecessary. Section VII also includes 
the mechanism for exploiting the temporal structure of the desired signal to blind beamforming for partial time jamming. Finally, a set of simulations illustrating the advantages of using FDSS combined with spatial diversity are provided in Section VIII.

\section{SPREAD SPECTRUM AND FREQUENCY DiVERSITY}

Direct sequence and frequency hopping are the most familiar techniques, among those that use spread spectrum, to combat bandlimited Gaussian interferers. In both cases, the transmitted signal is controlled by properly coding the carrier waveform. The role of coding is to enlarge the transmission bandwidth either by fast keying in the time domain or/and in the frequency domain. However, the performance of FHSS is sensitive to partial band jamming [2]. In DSSS systems, high levels of partial band jamming may force the receiver to employ a whitening filter, together with a sequence detector to remove inter-symbol interference (ISI) caused by the whitening stage. In addition to the highly undesired ISI, the interference excision filters increase the receiver complexity and give rise to self-noise, which is the induced correlation across the PN sequence. To mitigate the aforementioned problems, the FDSS system was proposed in [1], which encompasses the principles of the two systems DS and FH. The FDSS can be viewed as a DS scheme where successive chip frames, within a symbol, are transmitted in parallel over different nonoverlapping frequency bands. It is noted that the major difference between traditional FH communication systems and the system implementing FDSS is that in the later, the information symbol is repeated, coded or uncoded, in several frequency bands instead of using a single band at time. A simple mixture of both systems is to transmit the block of chip symbols of the first scheme simultaneously in different frequency bands with or without hopping. The flexibility of mixing the DS and FH schemes grows dramatically when a single information symbol produces the coded symbols through a channel encoder. Since all possible combinations are amenable to spatial diversity processing, the transmitted signal architecture depicted in Fig. 1 will be used to introduce the proposed spatial FDSS scheme. This architecture, which has been used by several authors (see [5], for example) to encompass all existing SS techniques, allows the presentation of the different alternatives offered by channel encoding, waveform coding, and frequency diversit to spread the spectrum of the transmitted signal.

The first stage of the scheme in Fig. 1 contains the channel encoder. Within this block, the information symbol is converted into coded symbols. The coded symbols are packed in blocks of $N_{1}$ symbols, and these blocks are arranged in $N$ branches. Without loss of generality, it is assumed that all the bands have the same bandwidth. In this case, the bandwidth is controlled by the number of branches $N$ as well as by the number $N_{1}$ of coded symbols per branch. This presents the basic contribution of the channel encoder to the bandwidth structure of the transmitted signal. When the number of symbols per branch is equal to one and the coded symbols are presented in the $N$ channels at the information symbol rate, there will still be a coding gain

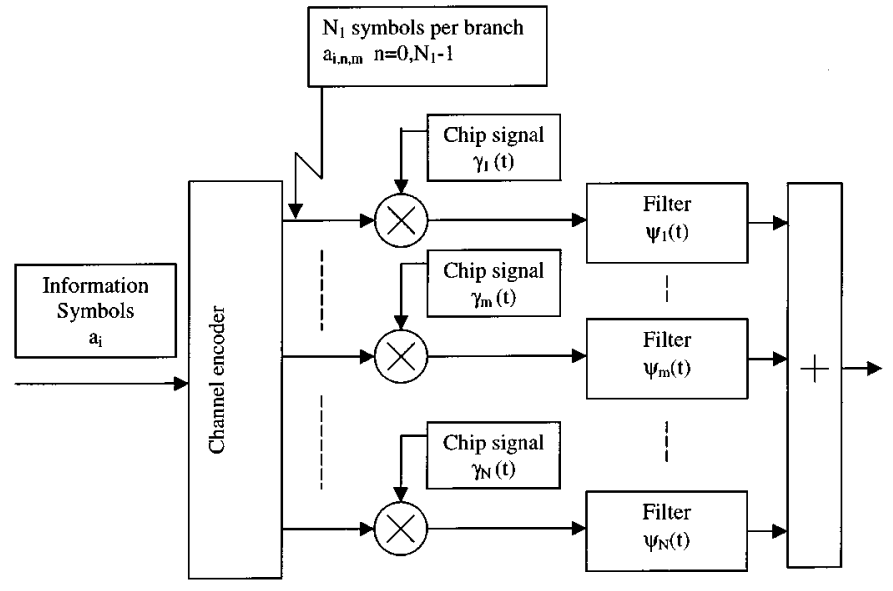

Fig. 1. General transmitter scheme for spread spectrum digital communications.

at the receiver by only using a pure repetition code. This type of coding amounts to the lowest complexity case, i.e., $N_{1}$ is equal to one, and the information symbol is presented simultaneously to all branches [pure repetition code $(N, 1)]$.

The second stage is the waveform coding. Every branch is multiplied by different, or equal, chip signal $\gamma_{m}(t)(m=1, N)$. The chip period $\tau_{c}$, as a fraction of the coded symbols rate $T_{s}$, determines the bandwidth for every branch. A special case is when the chip rate and the symbol rate coincide. In this case, there is a single chip symbol $\gamma_{i, m}$ per branch, and no spreading is produced. Furthermore, when the alphabet for the chip symbols is $(0,1)$, the transmitted signal can be viewed as associated with a frequency hopping system. In fact, traditional (single band) $\mathrm{FH}$, within this framework, requires only one chip to be on per a symbol interval, i.e., a single branch is activated at a time.

The third stage, which is the last in this sequence, contains the pulse shaping. The shaping functions $\psi_{i}(t)$ have different frequency supports. Multicarrier CDMA or OFDM can be encompassed in the proposed scheme, depending on the design of this last stage in the overall structure of signal generation. Assuming nonoverlapping frequency support for the shaping functions produces a transmitted signal that is usually referred to as frequency diversity spread spectrum signal. The nonoverlapping frequency bands often have the same bandwidth $B_{0}$. This bandwidth, which includes the roll-off factor, has to be the expected minimum jammer signal bandwidth. This choice guarantees that no narrowband intereferer is present for every frequency diversity band; otherwise, a whitening filtering, together with a Viterbi detector, will be needed to remove the ISI. The filter bank is implemented as a polyphase filter [6].

Finally, note that within an FDSS band, the system may have independent frequency hopping [4], allowing phase continuity and coherent optimal combining of the received replicas.

The formulation of the transmitted signal is shown in (1) for the general case when code, time, and frequency diversity are used simultaneously to spread the spectrum. In (1)

$N_{c} \quad$ number of chips per coded symbol interval;

$T_{s} \quad$ symbol duration; 
$N_{1} \quad$ number of coded symbols in every branch per information symbol;

$\tau_{c} \quad$ chip duration

$$
\begin{aligned}
x_{r}(t)= & \sum_{i} \sum_{m=1}^{N} \sum_{n=0}^{N_{1}-1} \sum_{l=0}^{N_{c}-1} a_{i, n, m} \gamma_{i, n, m, l} \\
& \cdot \Psi_{m}\left(\frac{t-i T_{s}-n\left(T_{s} / N_{1}\right)-l \tau_{c}}{T_{s} /\left(N_{1} \cdot N_{c}\right)}\right) .
\end{aligned}
$$

In the case of no spreading, $N_{1}=1$, and (1) simplifies to (2)

$$
x_{r}(t)=\sum_{i} \sum_{m=1}^{N} \sum_{l=0}^{N_{c}-1} a_{i, m} \gamma_{i, m, l} \Psi_{m}\left(\frac{t-i T_{s}-l \tau_{c}}{T_{s} / N_{c}}\right) \text {. }
$$

Finally, for a single chip per symbol interval and the repetition code, the transmitted signal is

$$
x_{r}(t)=\sum_{i} \sum_{m=1}^{N} a_{i} \gamma_{i, m} \Psi_{m}\left(\frac{t-i T_{s}}{T_{s}}\right) .
$$

This signal, which is referred to as FDSS signal, will be used from now on without loss of generality.

\section{OPTIMUM RECEIVER FOR FDSS}

This section highlights the differences between optimum and suboptimum detection in terms of performance versus complexity. It includes results from [1] that are most pertinent to the underlying problem and serve the derivation of the optimum spatial diversity receiver in the following sections.

The decision variable $\Lambda_{i}$ is a sufficient statistic to perform optimum symbol by symbol detection. This decision variable contains the following terms: the received samples, after matched filtering and sampling at the symbol rate $z_{i, m}$; the chip symbol, which is assumed to be known by the receiver $\gamma_{i, m}$; and the factors $F_{m}$, which depend on the spectral density of the noise plus the jammer in every frequency band $m$

$$
\Lambda_{i}=\sum_{m=1}^{N} z_{i, m} \gamma_{i, m}^{*} F_{m} .
$$

Since the bandwidth of every band is equal or less than the jammer's bandwidth, the jammer and the noise are assumed to have flat spectral densities, which are given by $J_{o}$ and $N_{o}$, respectively. In this case, the factor $F_{m}$ in the above equation is given by

$$
\begin{aligned}
& F_{m}=\frac{1}{\left(1+\left[\frac{J_{o}}{N_{o}}\right]\right)} \text { for bands hit by the jammer } \\
& F_{m}=1 \text { for free bands. }
\end{aligned}
$$

The decision for the received symbol $\hat{a}_{i}$ is the alphabet symbol $a_{i}$ that maximizes $\operatorname{Re}\left(a_{i}, \Lambda_{i}\right)$, where $\operatorname{Re}(\cdot)$ indicates the real part of a complex argument. The resulting probability of a symbol error is

$$
P_{e}=Q\left(\sqrt{\left(\frac{2 E_{s}}{N_{o}}\right) \beta}\right)
$$

where

$E_{s} \quad$ received energy per symbol;

$Q(\cdot)$ error function;

$\beta \quad$ loss factor that reflects the degradation due to the presence of jammer.

This factor depends only on the spectral density of the jammer, normalized by the noise density, as well as the number of hit and free bands (which are denoted by $N_{h}$ and $N_{f}$, respectively) relative to the total number of frequency bands $N$

$$
\beta=\frac{1}{N}\left\{\sum_{N_{h}} \frac{1}{1+\frac{J_{o}}{N_{o}}}+\sum_{N_{f}} 1\right\} .
$$

The above factor can be modified accordingly in the case of unequal jammer strength for every band. When the jammer has the same strength in all the hit bands, the above formula can be expressed as a function of the fraction of hit bands $\eta$. This factor is the key parameter in the performance of FDSS systems [3]

$$
\beta=\left\{\left(\frac{\eta}{\left(1+\left(\frac{J_{o}}{N_{o}}\right)\right)}\right)+(1-\eta)\right\} .
$$

A suboptimum receiver does not use the side information corresponding to the jammer strength, and the decision variable $\Lambda_{i}$ is only formed by the free bands. The probability of symbol error in such a case is given by (9), which shows that the loss factor $\beta$ is equal to $1-\eta$ because it is independent of the jammer strength

$$
P_{e}=Q\left(\sqrt{\left(\frac{2 E_{s}}{N_{o}}\right)(1-\eta)}\right) .
$$

Note that both receivers degrade in performance when $\eta$ gets close to one, that is, when the jammer spreads its bandwidth covering the entire transmission band. A detailed study of both receivers and their comparison with DS and FH systems can be found in [1], [2], and [4]. This study shows, in many respects, the advantages of FDSS in partial jamming suppression over traditional DSSS and FHSS systems. It should be noted that for a DSSS, the processing gain is, in essence, $1 / \eta$, and the equivalent $\beta$ is given by

$$
\beta_{D S}=\left(\frac{1}{1+\eta\left(\frac{J_{o}}{N_{o}}\right)}\right)
$$

which is always lower than that of the FDSS optimum receiver.

Spatial diversity in FDSS is next introduced to alleviate, whenever it is possible, the undesired impact of the hit bands on the receiver performance. This is to say that spatial processing strives to reduce the effective $\eta$ by removing jammer contamination in the hit bands.

\section{SPATIAL PROCESSING IN FDSS SYSTEMS}

As mentioned in the previous section, when the number of bands hit by the interference is close to the total number of fre- 
quency bands $N$, both the optimum and suboptimum receivers degrade in performance. Furthermore, high levels of jamming tend to impair the performance of both receivers, rendering all efforts of labeling every band useless. As it is typical in digital transmission, when temporal processing is insufficient to counteract interferences, spatial diversity may be introduced to remove, or at least reduce, the jammer contamination of the desired signal, independent of its temporal characteristics. Taking advantage of the frequency diversity available in FDSS systems, we propose a procedure to design optimum spatial diversity processing that is blind to the desired signal waveform. In fact, the procedure is valid for all the SS schemes that implement the architecture of Fig. 1 for signal generation and transmission, provided that $N$ is greater than one.

It is assumed that the desired signal is present in all frequency bands. It is also assumed that the labeling of the hit bands is properly done (This assumption is latter relaxed). All the air interfaces, down conversion, and filtering channels are calibrated. Full coherence is assumed for the desired signal across spatial diversity channels.

Let us assume that $\underline{X}_{f, n}$ and $\underline{X}_{h, n}$ are the snapshots, at time $n$, of the free and hit bands, respectively. Both bands are selected to start up the design of the respective optimum spatial combiners $\underline{w}_{f}$ and $\underline{w}_{h}$. Once the chip symbol is removed for the two bands selected, i.e., $\underline{X}_{f, n} \Rightarrow \gamma_{f, n}^{*} \underline{X}_{f, n}$ and $\underline{X}_{h, n} \Rightarrow$ $\gamma_{h, n}^{*} \underline{X}_{h, n}$, the desired signal, which is present in both bands but more pronounced in the free bands, can be used as the reference signal. The design of both combiners is performed through the quadratic cost function minimization

$$
\xi=E\left\{\left|\underline{w}_{f}^{H} \underline{X}_{f, n}-\underline{w}_{h}^{H} \underline{X}_{h, n}\right|^{2}\right\} .
$$

The undesired solutions to the above objective function are either of the null vector for both combiners, which represent the case when both the desired and the jammer signals are nulled out simultaneously. A suitable technique to avoid both solutions is to set the cross correlation of both combiner's outputs to some chosen value

$$
\operatorname{Re}\left\{E\left[\underline{w}_{f}^{H} \underline{X}_{f, n} \underline{X}_{h, n}^{H} \underline{w}_{h}\right]\right\}=\phi
$$

where $\phi$ is some constant different from zero. Naming the array autocovariances and cross covariance of the two frequency channels as

$$
\begin{aligned}
\underline{\underline{R}}_{f} & =E\left[\underline{X}_{f, n} \underline{X}_{f, n}^{H}\right] \\
\underline{\underline{R}}_{h} & =E\left[\underline{X}_{h, n} \underline{X}_{h, n}^{H}\right] \\
\underline{\underline{P}} f, h & =E\left[\underline{X}_{f, n} \underline{X}_{h, n}^{H}\right]
\end{aligned}
$$

the minimization problem becomes

$$
\begin{aligned}
\text { Minimize } \xi= & \underline{w}_{f}^{H} \underline{\underline{R}}_{f} \underline{w}_{f}+\underline{w}_{h}^{H} \underline{\underline{R}}_{h} \underline{w}_{h}-\underline{w}_{f}^{H} \underline{\underline{P}}_{f, h} \underline{w}_{h} \\
& -\underline{w}_{h}^{H} \underline{\underline{P}}_{f, h}^{H} \underline{w}_{f}
\end{aligned}
$$

constrained to

$$
\underline{w}_{f}^{H} \underline{\underline{P}}_{f, h} \underline{w}_{h}+\underline{w}_{h}^{H} \underline{\underline{P}}_{f, h}^{H} \underline{\underline{w}}_{f}=\phi .
$$

Forming the Lagrangian and setting partial derivatives equal to zero, the optimum spatial combiners are obtained as

$$
\begin{aligned}
\underline{\underline{R}}_{f} \underline{w}_{f} & =(1+\lambda)^{2} \underline{\underline{P}}_{f, h} \underline{\underline{R}}_{h}^{-1} \underline{\underline{P}}_{f, h}^{H} \underline{\underline{w}}_{f} \\
\underline{w}_{h} & =(1+\lambda) \underline{\underline{R}}_{h}^{-1} \underline{\underline{P}}_{f, h}^{H} \underline{w}_{f}
\end{aligned}
$$

where $\lambda$ is the Lagrange multiplier. Since $\xi$ is minimum for minimum $\lambda$, the optimum combiner for the jammer-free band is the eigenvector of (15a) associated with the minimum eigenvalue. After $\underline{w}_{f}$ is found, the combiner for the hit band is just the Wiener solution for the cross correlation vector $\underline{\underline{P}}_{f, h}^{H} \cdot \underline{w}_{f}$. Once the combiner of the free band is derived, the rest of the combiners can be derived by using the band free output as a time reference for all other bands. The unconstrained minimization procedure is posed in (16a), and the respective solution for the combiners is given in (16b). Since every frequency band has to be calibrated in the front end, the reference combiner is normalized for response equal to one in a preselected diversity channel (i.e., $\left.w_{f}(1)=1\right)$ before it is used in $(16 \mathrm{~b})$; at the same time, this normalization also helps the weights implementation with finite register length

$$
E\left[\left|\underline{w}_{f}^{H} \underline{X}_{n, f}-\underline{w}_{h}^{H} \underline{X}_{n, h}\right|^{2}\right]_{\min } \quad \text { for } h \neq f
$$

and

$$
\underline{w}_{h}=\underline{\underline{R}}_{h}^{-1} \underline{\underline{P}}_{h}, f \underline{w}_{f} .
$$

It is noteworthy that since the jammer is uncorrelated from one band to another due to the chip demodulation, any two bands can be selected to start up the design in (16a). Once a combiner is derived, its output provides a time reference for the rest of the bands. This implies that labeling is not essential to design the spatial processor. Nevertheless, when required, a suitable procedure to determine the free and the hit bands to be used in (15a) is to choose the two bands with minimum and maximum received power, respectively.

Note that when the transmission bandwidth is very small compared with the central frequency and adequate labeling of free and hit bands is available, there is a great saving in computations since the combiners derived in (15), for a pair of free and hit bands, can be applied directly in the rest of the bands.

The above optimum solution holds close resemblance to the one obtained using the cross self-coherence restoral (SCORE) algorithm derived in [7]. In the Score algorithm, it is assumed that the desired signal is spectrally self coherent at frequency $f_{o}$ if the correlation between the desired signal $s(t)$ and $s(t)$ frequency shifted by $f_{o}$ is nonzero for some lag $\tau$. In the underlying frequency diversity spread spectrum problem, the frequency shift represents the offset in the carrier frequency between two replicas of the signal frequency band, and the lag variable $\tau$ takes a zero value. In [7], however, the quadratic cost function minimization is replaced by the maximization of the strength of the cross correlation coefficient between the outputs of two beamformers.

Some of the key differences of the two techniques are the following. 
1) In the spatial diversity FDSS method, the exact values of $f_{o}$ and $\tau$ are known a priori and do not depend on modulation schemes.

2) Adopting the same self-coherent terminology used in [7], the proposed spatial diversity FDSS technique, through its frequency replicas, provides, in essence, not one but multiple sets of the time-lag and frequency-lag $\left(\tau, f_{o}\right)$ self-coherent parameters. These sets can be used in a combined multichannel-based processing for enhanced performance.

3) Unlike the technique in [7], the data snapshots for some frequency bands in the spatial diversity FDSS systems are free of the jammer signal; this is a property that is fully utilized in this paper if proper labeling of the bands is provided.

To gain insight into the design method, we inspect the design equation for the case when the desired signal is a point source. In this case, the cross covariance matrix is rank one

$$
\underline{\underline{P}}_{f, h}=\kappa_{O} \underline{P}_{f} \underline{P}_{h}^{H}
$$

and the corresponding equations for the optimal combiners reduce to

$$
\underline{w}_{f} \propto \underline{\underline{R}}_{f}^{-1} \underline{P}_{f}
$$

and

$$
\underline{w}_{h} \propto \underline{\underline{R}}_{h}^{-1} \underline{P}_{h}
$$

as expected from the optimum combiner equations, when the vectors of the second term of (17) are known. Nevertheless, the rank-one approximation of the cross covariance provides poor performance when the number of snapshots used in its estimation is small. In general, the formulation (15) offers greatly improved results over the outlined rank-one approximation procedure. Further, the rank-one procedure requires the point source model for the desired signal, which, in general, is not typically the case in radio communication systems.

\section{OPTIMUM DeteCtion AND CROSS-Over}

It is often the case that the effect of the spatial processing on subsequent stages in the receiver is ignored. In the underlying problem, it is important to include such an effect specifically when the spatial combiner varies from a frequency channel to another for both the suboptimum and optimum receivers. The spatial combiner does not change the statistics and the structure of the likelihood function. Only the changes in the power of both the desired signal and the jammer, as they move through the spatial structures, alter the sufficient statistic for optimum detection. The sufficient statistic for optimum detection depends on the combiner response to all sources impinging the aperture and is given by

$$
\Lambda_{i}=\sum_{m=1}^{N} z_{m, i}\left(\frac{w_{m d}^{*}}{J_{o}\left|w_{m j}\right|^{2}+N_{o}\left|\underline{w}_{m}\right|^{2}}\right)
$$

where $z_{m, i}$ is the combiner output of the frequency channel $m$ at symbol $i$ after chip despreading and matched filtering, and scalars (i.e., $w_{m d}=w_{m}^{H} S_{d}$ being $S_{d}$ the steering of the desired for point source scenarios) $w_{m d}$ and $w_{m j}$ are the responses of the combiner $w_{m}$ to the desired signal and to the jammer, respectively.

From (19), it is clear that the response of the combiners is required in order to determine the sufficient statistics for optimum detection. Furthermore, it may occur that the spatial signature of the jammer completely mask the desired signal. This is the case when the jammer crosses over the DOA of the desired source in an optic channel. In consequence, it is necessary to keep control of the adequate labeling at the combiners' outputs. From the three values involved in each term in (19), only the norm of the combiner is directly available to the designer; the other two must be estimated from data. It can readily be shown that by taking the free band in (15a) as a reference band, the factor $F_{n}$ for the rest of the bands is given by

$$
F_{m}=\left(\frac{\left(\frac{w_{m d}}{w_{f d}}\right)^{*}}{\left(\frac{J_{o}\left|w_{m j}\right|^{2}+N_{o}\left|\underline{w}_{m}\right|^{2}}{N_{o}\left|\underline{w}_{f}\right|^{2}}\right)}\right) ; \quad \forall m \neq f
$$

and using this factor the sufficient statistic

$$
\Lambda_{i}=\frac{w_{f d}}{N_{o}\left|\underline{w}_{f}\right|}\left[z_{f, i}+\sum_{\substack{m=1 \\ m \neq f}}^{N} z_{m, i} F_{m}\right] .
$$

The term corresponding to the spectral density of the noise $N_{o}$ can be determined from the noise eigenvalues of $\underline{\underline{R}}_{f}$. The numerator in the above equation can be provided using the identity

$$
\left(\frac{w_{m d}}{w_{f d}}\right)^{*} \approx \frac{\underline{w}_{f}^{H} \underline{\underline{P}}_{f m} \underline{\underline{w}}_{m}}{\underline{\underline{w}}_{f}^{H} \underline{\underline{R}}_{f} \underline{w}_{f}} .
$$

As for the estimation of the denominator in (20), we use two key formulations of the design criterion for the combiners. The first expression is the MSE incorporating (16b)

$$
\operatorname{MSE}(m)=\underline{w}_{f}^{H}\left(\underline{\underline{R}}_{f}-\underline{\underline{P}}_{m f}^{H} \underline{\underline{R}}_{m}^{-1} \underline{\underline{P}}_{m f}\right) \cdot \underline{w}_{f}
$$

and the second relationship comes from the definition of the minimization criterion. The MSE is lower bounded by the residuals levels of jammer and noise

$$
\operatorname{MSE}(m) J_{o} \geq\left|w_{m j}\right|^{2}+N_{o}\left(\left|\underline{w}_{m}\right|^{2}+\left|\underline{w}_{f}\right|^{2}\right) .
$$

The equality holds for the case where the jammer does not shadow the desired signal and it is properly cancelled in the MSE. Thus, using (22)-(24) in (20), we obtain an estimate of the factor to be used in the decision variable for the bands different from the reference band

$$
F_{m}=\frac{\frac{\underline{\underline{w}}_{m}^{H} \underline{\underline{P}}_{m f} \underline{\underline{w}}_{f}}{\underline{w}_{f}^{H} \underline{\underline{R}}_{f} \underline{w}_{f}}}{\left(\left[\frac{\mathrm{MSE}(m)}{N_{o}\left|\underline{w}_{f}\right|^{2}}\right]-1\right)} .
$$

Note that this factor is smaller than the optimum weighting since the inequality (24) is used to estimate the denominator. 
This factor accounts for the residual loss from the spatial diversity processing due to the presence of the jammer. In the case where the desired source is completely shadowed by the jammer, the factor $F_{n}$ depicts negligible contribution of the hit bands to the decision variable when compared with the terms of the bands that are free of the jammer.

\section{ADAPTIVE DESIGN}

This section describes an adaptive algorithm to the constrained minimization described in Section IV. It should be emphasized that the fundamental step is to find the response of the free-band combiner $\underline{w}_{f}$. Once this combiner is found at each iteration, the update of the rest of the combiners, including $\underline{w}_{h}$, is done in a time reference combining framework, where the output of $\underline{w}_{f}$ acts as the reference waveform. The adaptive algorithm to be presented herein can be viewed as an improved version of the so-called maximin algorithm reported in [8] to maximize the quotient of two quadratic forms. As stated in Section IV, the presence of chip symbol modulation decorrelates the jammer from one band to another. Under this premise, the band selection is no longer crucial in the resulting performance of the method.

The basic update of the selected beamformers is done with the instantaneous gradient of the Lagrange objective $\xi$

$$
\begin{aligned}
\xi & =\left|y_{f, n}-y_{h, n}\right|^{2}-\left(\lambda_{n}-1\right) \cdot 2 \cdot \operatorname{Re}\left[y_{f, n} \cdot y_{h, n}^{*}\right] \\
& =\left|y_{f, n}-y_{h, n}\right|^{2}-\left(\lambda_{n}-1\right) \phi_{n}
\end{aligned}
$$

where $\left(\lambda_{n}-1\right)$ is the Lagrange multiplier, and

$$
\begin{aligned}
& y_{f, n}=\underline{w}_{f, n}^{H} \underline{X}_{f, n} \\
& y_{h, n}=\underline{w}_{h, n}^{H} \underline{X}_{h, n}
\end{aligned}
$$

are the combiners' output. The update equations are then given by

$$
\begin{aligned}
& \underline{w}_{f, n+1}=\underline{w}_{f, n}-\mu_{f, n} \underline{X}_{f, n}\left(y_{f, n}^{*}-\lambda_{n} y_{h, n}^{*}\right) \\
& \underline{w}_{h, n+1}=\underline{w}_{h, n}-\mu_{h, n} \underline{X}_{h, n}\left(y_{h, n}^{*}-\lambda_{n} y_{f, n}^{*}\right) .
\end{aligned}
$$

The Lagrange multiplier has to be set such that the new weights, prepared for snapshot $n+1$, hold the constraint at snapshot $n$. In other words, the updated combiners have to satisfy

$$
\phi_{n}=2 \cdot \operatorname{Re}\left[\underline{w}_{f, n+1}^{H} \underline{X}_{f, n} \underline{X}_{h, n}^{H} \underline{w}_{h, n+1}\right] .
$$

The step-size parameters are set to

$$
\mu_{f, n}=\frac{\alpha}{W_{f, n}} ; \quad \mu_{h, n}=\frac{\alpha}{W_{h, n}} .
$$

The denominators in the above equation are the snapshot powers defined by

$$
\begin{aligned}
& W_{f, n}=\left|\underline{X}_{f, n}^{H} \underline{X}_{f, n}\right| \\
& W_{h, n}=\left|\underline{X}_{h, n}^{H} \underline{X}_{h, n}\right| .
\end{aligned}
$$
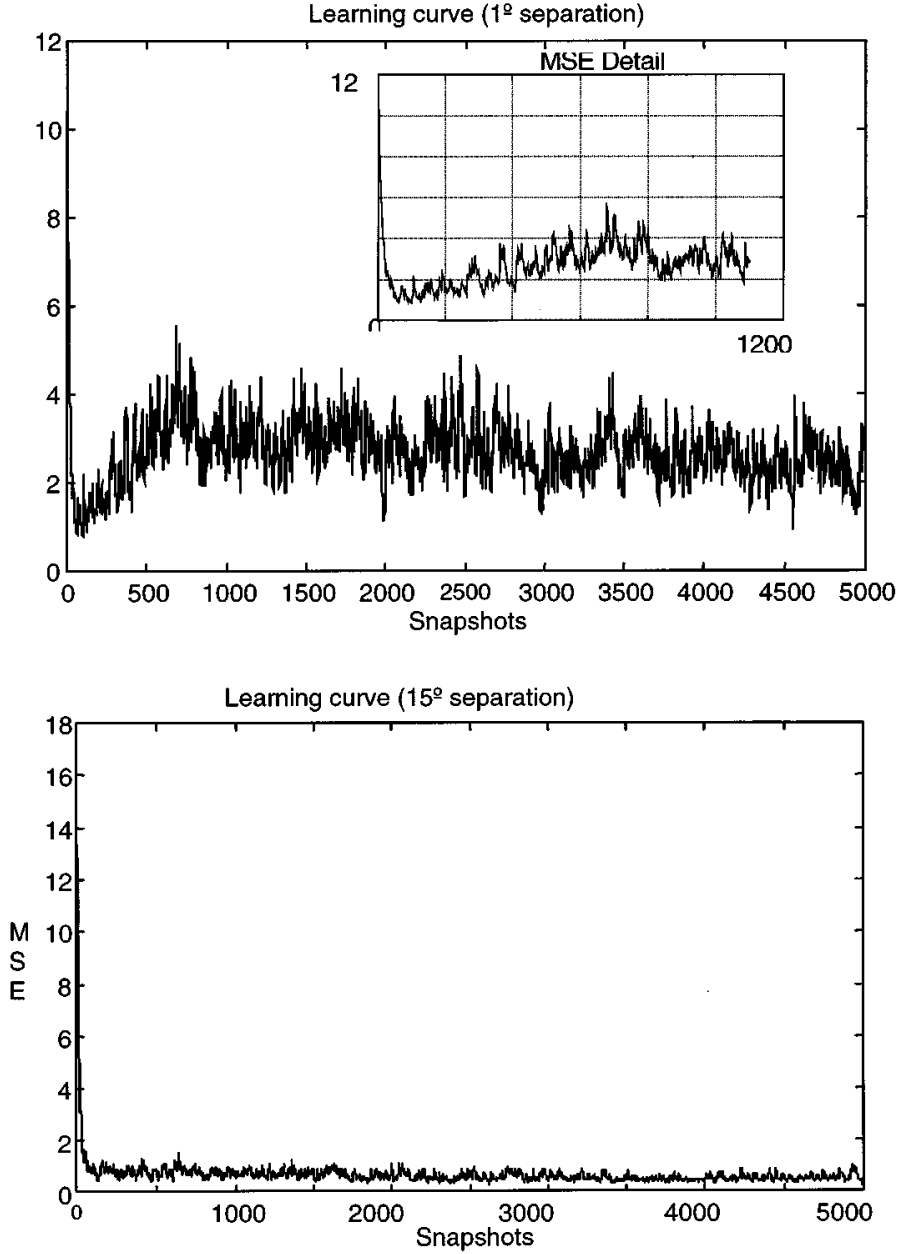

Fig. 2. Learning curve for the adaptive algorithm, i.e., the smoothed MSE versus snapshots used, for DOA separations of $1^{\circ}$ (top) and $15^{\circ}$ (bottom) between the desired signal and the jammer. The scenario and algorithm parameters are six bands, $50 \%$ bands jammed, 1 and $10 \mathrm{~dB}$ of SNR for desired signal and jammer, respectively, $\alpha=0.01, \beta=0.9$, and five sensors half wavelength ULA array.

Including the weight updates in (29) and assuming that the past weights satisfy the constraint, the following equation provides the selection for $\lambda_{n}$ :

$$
\frac{1}{2} \lambda_{n}^{2} \phi_{n} \alpha+\lambda_{n}\left(P_{f, n}+P_{h, n}\right)(1-\alpha)+\phi_{n}\left(\frac{\alpha}{2}-1\right)=0
$$

where

$$
\begin{aligned}
& P_{f, n}=\left|\underline{w}_{f, n}^{H} \underline{X}_{f, n}\right|^{2} \\
& P_{h, n}=\left|\underline{w}_{h, n}^{H} \underline{X}_{h, n}\right|^{2} .
\end{aligned}
$$

Since the parameter $\alpha$ has to be set equal to small values, which are usually below 0.02 , to obtain reasonable levels of misadjusment, the terms including $\alpha$ in (32) can be neglected in favor of the other. This results in

$$
\lambda_{n}=\frac{\phi_{n}}{\left(P_{h, n}+P_{f, n}\right)} .
$$

Since the constraint acts like an automatic control gain for the system, the numerator of the above formula can be set to a constant value. Note that this constant will be, on the average, the power at the output of the free band. This value was set to one in 
the simulations included in this section. It is interesting to note that at the steady state, as the output powers tend to be equal, the Lagrange multiplier will tend to one. On the other hand, at the start up, when $P_{f}$ and $P_{h}$ are expected to be different, the multiplier will be smaller than one. In other words, the Lagrange multiplier acts like a flag, indicating to the updating algorithm how both output signals have to be compared to provide a proper error correction.

A realistic setup of the algorithm demands some smoothing of the instantaneous powers defined previously. The resulting performance, in terms of convergence rate, does not show high sensitivity to this parameter, providing it is above a threshold. For different scenarios, the values of the smoothing factor that are above 0.8 have shown small impact in the learning curve.

Fig. 2 depicts the learning curve (i.e., the smoothed MSE versus number of snapshots) for two different scenarios, which differ in the angular separation between the desired source and the jammer. The rest of the parameters are FDSS of six bands, $\eta$ equal to $50 \%$, five sensors half wavelength ULA array, the desired and jammer signals being 1 and $10 \mathrm{~dB}$, respectively, $\alpha=0.01$, and $\beta=0.9$. From this figure, it is clear that the adaptive algorithm exhibits good behavior, which makes it a candidate alternative to frame processing method suitable for TDMA, which has been reported in Section IV.

\section{COMmEnTS AND ExTENSIONS OF THE PROCEDURE}

It is important to note that the proposed method is based merely on the coded structure of the desired signal in the frequency domain. We should stress, however, that the applicability of this method is granted, regardless of the nature of the code, i.e., whether it is being pure repetitive or otherwise. With the same perspective, it is easy to realize that as long as the desired signal has a structure (we refer to "a structure" as any repetition over any diversity axis like code, frequency, time, etc.), optimum spatial processing is attainable.

From the above perspective, spatial processing methods, which is referred to as blind, are not entirely blind, since some information concerning the signal structure is used at the receiver to obtain optimum processing. In communications systems, this structure is provided for proper matching between modulation and demodulation schemes at both ends of the communications channels.

Another way to describe the underlying advantages of FD systems for blind beamforming is to translate the signal structure to the property of exact prediction. The structure of the FDSS transmitted signal, in essence, has allowed the desired signal to be exactly predicted from one band to another and thereby serves as a reference. Since this scheme uses predictability in the frequency domain, it is evident that some correspondence has to exist in the time domain. This possibility was outlined in Section II, when it was proposed to divide the chip signal in several segments within a symbol and to transmit every slot in a different frequency band. The time domain dual is the transmission of these slots sequentially in time (time diversity) to counteract partial time

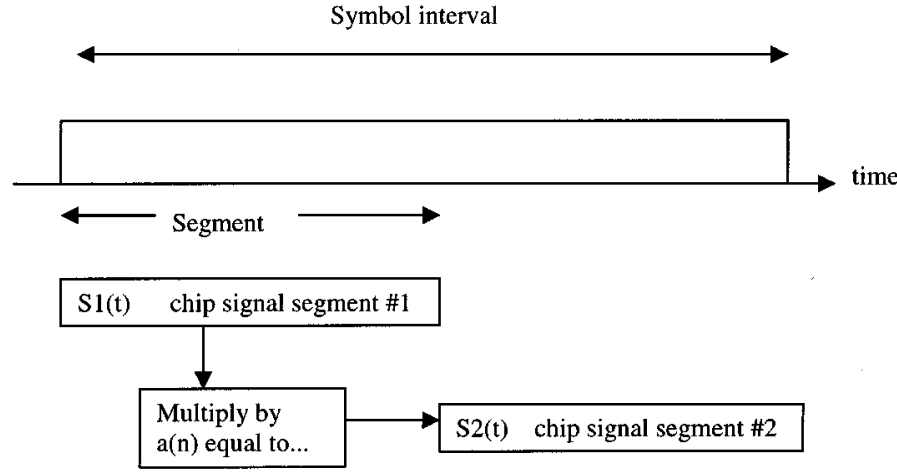

Fig. 3. Code structure within time diversity. A segment of the desired signal can be exactly predicted from the previous segment.

jamming [9]. With time diversity, the segments within an information symbol can be predicted and used to form the blind combiner. For clarity of the presentation, let us assume that the chip signal within a symbol is divided in two segments, as indicated in Fig. 3. Note that the pure repetition code is also included within this general scheme (i.e., the same chip code is used twice per symbol interval).

Since the described structure in the time domain is exactly the same as that of the FDSS in the frequency domain, the optimum spatial combiner for the corresponding desired signal can be derived from the minimization of $\xi$ defined as

$$
\xi=E\left\{\left|\underline{w}_{f}^{H} \underline{X}_{n, 1}-a(n) \underline{w}_{h}^{H} \underline{X}_{n, 2}\right|^{2}\right\}
$$

where $X_{n, 1}$ are the snapshots corresponding to segments one, and $X_{n, 2}$ are the snapshots corresponding to segment two. This formulation is adequate for partial time jamming as the desired signal temporal structure allows exact prediction. More interesting is the case where the minimization of (35) is carried over a single combiner, which is possible if the jammer remains uncorrelated from time segment to segment

$$
\xi=E\left\{\left|\underline{w}^{H} \underline{X}_{n, 1}-a(n) \underline{w}^{H} \underline{X}_{n, 2}\right|^{2}\right\}
$$

Since the jammer and the noise are assumed uncorrelated for the two segments selected, the optimum combiner will select only the signal that is exactly predicted by $a(n)$, i.e., the desired signal. Of course, the additional constraint used for FDSS has to be added in order to avoid the trivial solution to the minimization of (36). The exact prediction required for the procedure, which was outlined previously, can be also provided by the channel encoder. Note that using the same combiner precludes optimal detection but reduces the complexity in the suboptimum detector.

For further illustration of the notion of signal prediction and diversity, we selected below the case of an unmodulated carrier at baseband frequency $f_{0}$. Assuming that the maximum time for coherence in the jammers is smaller than $t_{o}$ (which is the case when $t_{o}$ is greater than the inverse of the interferer's symbol rate), the minimization objective is

$$
\xi=E\left\{\left|\underline{w}^{H} \underline{X}_{n}-\exp \left(-j 2 \pi f_{o} t_{o}\right) \underline{w}^{H} \underline{X}_{n+t_{o}}\right|^{2}\right\} .
$$


This objective, which is constrained by the nonzero value of the cross correlation between the two outputs, produces adequate nulling of the jammer at the combiners' output. Defining

$$
\underline{\underline{R}}=E\left(\underline{X}_{n} \underline{X}_{n}^{H}\right), \underline{\underline{P}}=E\left(\underline{X}_{n} \underline{X}_{n+t_{o}}{ }^{H}\right) \exp \left(-j 2 \pi f_{o} t_{o}\right)
$$

the optimum combiner is the eigenvector associated with the minimum eigenvalue

$$
\underline{\underline{R} w}=\lambda\left(\frac{\underline{\underline{P}}+\underline{\underline{P}}^{H}}{2}\right) \underline{w} .
$$

Fig. 4 shows an example using the exact prediction property of an unmodulated carrier. The top part of this figure shows the beamformer response (top), whereas the bottom part depicts the beamforming scheme, which reflects the low complexity of the reference loop. The scenario contains two BPSK interferers (10 and $3 \mathrm{~dB}$ of SNR) at symbol rate greater than $t_{o}^{-1}$ with DOA's equal to 0 and $10^{\circ}$, together with the desired unmodulated carrier $(0 \mathrm{~dB})$ impinging from $-20^{\circ}$. The baseband frequency is 0.25 for all sources. The adequate response of the combiner designed from 500 snapshots (250 each set), using the aforementioned procedure, is clearly evident.

It is noteworthy that this system requires only the nominal frequency of the desired signal and does not need phase synchronism, which is very convenient in practice. This allows a great saving in complexity and threshold margin for very low SNRI's.

Finally, note that since frequency structure effectively removes partial band jamming, time structure does the same with partial time jamming. As a consequence, if the structure of the transmitted signal has, simultaneously, time and frequency diversity, the receiver will be robust against these two undesired jamming effects.

\section{SIMULATIONS}

The most important issue concerning the usefulness of spatial combining for frequency diversity is the adequate weighting of every term of the sum forming the decision variable $\Lambda_{m}$ in (19). This factor reflects the limitations of the combiner when the jammer shadows globally or partially some bands of the FD system. The estimate of this factor is proposed in (24), as is shown in Fig. 5, and presents this estimate for different DOA separation between the jammer and the desired signal. For every separation, a set of 400 snapshots has been used. The variation of the DOA separation was set from $0^{\circ}$ (complete shadowing) up to $20^{\circ}$. The SNR of the jammer was, in all cases, $10 \mathrm{~dB}$ (SJR $=-10$ $\mathrm{dB})$. No chip modulation has been used in the simulations; in consequence, the jammer is fully correlated from band to band, and the performance reported can be considered, in practice, to be a lower bound. The desired signal is BPSK modulated, and its power is changed from 10 down to 0 $\mathrm{dB}$ (step $1 \mathrm{~dB}$ ). The desired signal permanent location was set equal to $10^{\circ}$ from the broadside. The FDSS system has


Fig. 4. Beamforming response based on the exact prediction of the desired signal. Desired (unmodulated carrier) and jammers are labeled with lines in both plots on top. Bottom: the beamforming architecture with the reference loop.



Fig. 5. Shadowing factor $F_{n}$ as a function of the DOA separation of the desired versus the jammer for several SNR (SNRd) of the desired signal.

six frequency bands out of which bands 1,2 , and 4 are hit by the jammer $(\eta=50 \%)$ at $10 \mathrm{~dB}$ of jammer to noise ratio (JNR) each. The labeling of the free and hit bands was done from a power detector. The aperture was an ULA array of five sensors. The estimate of the shadowing factor $F_{n}$ improves when the SNR of the desired is high, that is, 

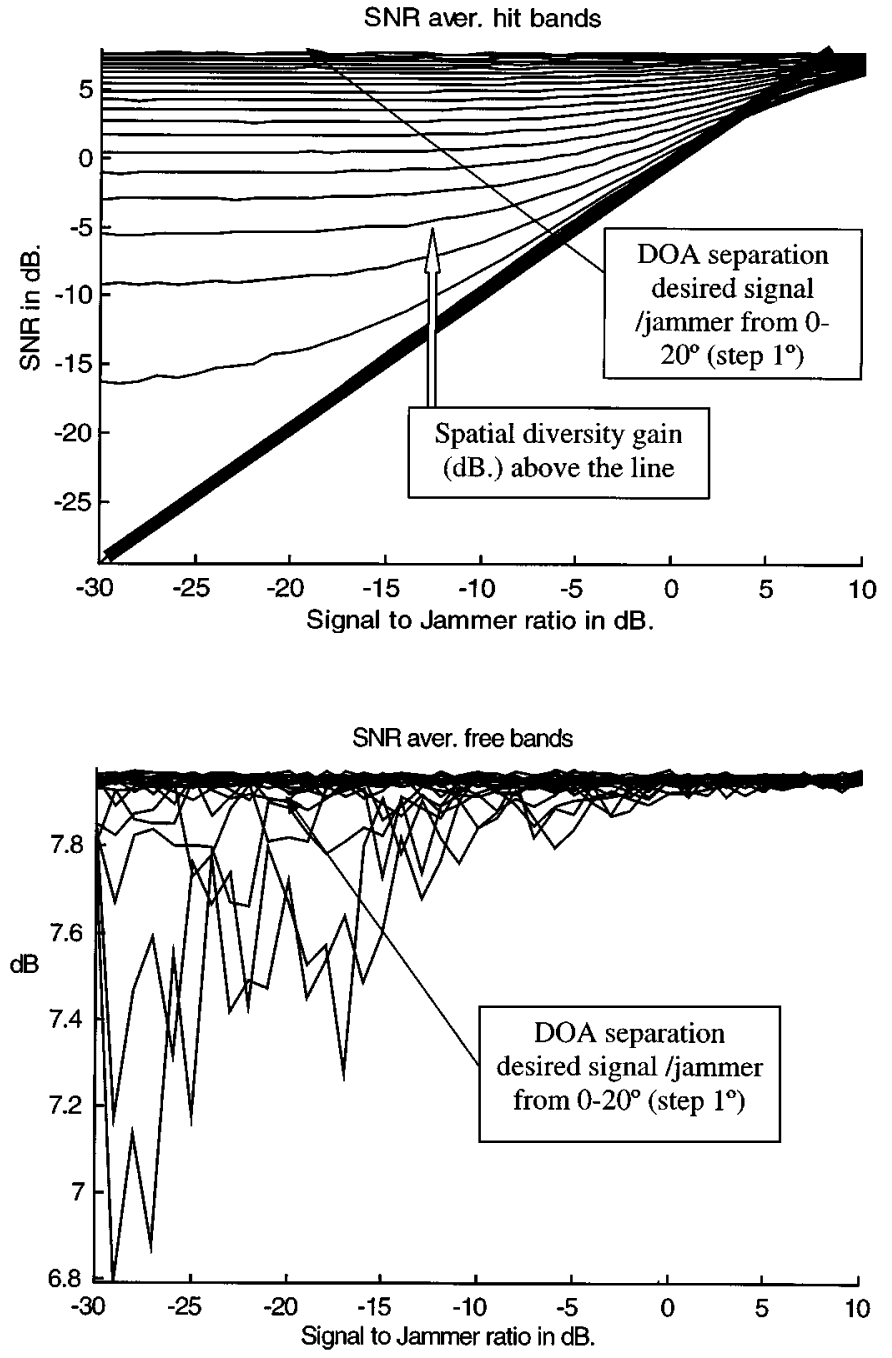

Fig. 6. Average actual SNR for the hit bands (top) and for the free bands (bottom) versus signal-to-jammer ratio (SJR) for different DOA separation between the desired source and the jammer.

$F_{n}$ is close to zero for zero degrees of source separation and close to one when the jammer is impinging on the aperture from secondary lobes (for this case, the spatial bandwidth of the aperture at the central frequency was $20^{\circ}$ ). Performance deterioration for low signal-to-noise ratio is expected since the difference between the free and the hit bands decreases with high levels of noise. When factor $F_{n}$ was used in the detector statistics, the bit error rate (BER) was always below or equal (complete shadowing) to the resulting one for the suboptimum detector [BER corresponding to SNRd plus the number of free bands $(10 . \log 3 \mathrm{~dB})$, plus the number of spatial diversity channels $(10 \log 5 \mathrm{~dB})]$.

It is also interesting to measure for the same scenario the change in the SNR over the different bands at the combiners outputs for different SJR's. Fig. 6 shows (top) for the hit bands and (bottom) for the free bands the average of the actual SNR for each group, together with the impact of the corresponding shadowing. The input SNRd is set to $1 \mathrm{~dB}$. It is important to note that the contribution of the SNR in the free bands increases with increased jammer power and with the shadowing effect. The worst case is represented by a $30-\mathrm{dB}$ jammer above the desired in every hit band and DOA equal to the same as desired signal. The result is $1 \mathrm{~dB}$ less than the case when there is two degrees of separation between the jammer and the desired signal. This plot provides evidence that the new front end is able to resort at any time to the suboptimum detector. This is because the free bands remain almost unaffected by the spatial combiner processing. The role of spatial processing for improving SNR in the hit bands should be well appreciated. This produces in an effective reduction in factor $\eta$, which, for this aperture, reduces to zero when the separation of the desired and the source is greater than $6^{\circ}$.

\section{CONCLUSIONS}

It has been shown that the use of diversity (basically frequency diversity), in the sense of several replicas of the desired signal, allows the optimum design of the spatial combiners that are blind to the source waveform. In a wide sense, it can be stated that whenever the desired signal along any diversity axis has an exact representation over diversity components, the spatial combiner can be optimally designed without additional diversity slots for a reference signal. The proposed design procedure minimizes the MSE between two selected diversity components, as in an exact prediction problem, with the constraint of a cross correlation factor different from zero. It is remarked that the diversity could be time, frequency, encode, or any mixture of them. The procedure is blind to the desired signal waveform. When the jammer signs in some or all diversity, the optimum detector demands for an estimate of this effect in order to properly adjust the likelihood in every diversity branch. To cope with this problem, an estimate has been derived from the design parameters. This estimate almost loads the detector to operate only on the jamming free diversity branches when the jammer shows strong presence. This is, in essence, an automatic convergence to the so-called suboptimum receiver. It has been outlined that whenever any kind diversity allows exact prediction of only the desired signal over two diversity components, blind spatial processing is always available.

\section{REFERENCES}

[1] G. K. Kaleh, "Frequency diversity spread spectrum communications system to counter bandlimited Gaussian intereference," IEEE Trans. Commun., vol. 44, pp. 886-893, July 1996.

[2] - "Performance comparison of frequency diversity and frequency hopping spread spectrum systems," IEEE Trans. Commun., vol. 45, pp. 910-912, Aug. 1997.

[3] J. G. Proakis, Digital Communications, 3rd ed. New York: Mc-Graw Hill, 1995, ch. 12-15.

[4] E. Lance and G. K. Kaleh, "A diversity scheme for a phase-coherent frequency-hopping spread spectrum system," IEEE Trans. Commun., vol. 45, pp. 1123-1129, Sept. 1997.

[5] R. Prasad and S. Hara, "An overview of multicarrier CDMA," in Proc. IEEE Fourth Int. Symp. Spread Spectrum Techn. Appl., Mainz, Germany, Dec. 1996, pp. 107-113.

[6] R. E. Crochiere and L. R. Rabiner, Multirate Digital Signal Processing. Englewood-Cliffs, NJ: Prentice-Hall, 1983.

[7] B. G. Agee, S. V. Schell, and W. A. Gardner, "Spectral self-coherence restoral: A new approach to blind adaptive signal extraction using antenna arrays," Proc. IEEE, vol. 78, pp. 753-767, Apr. 1990. 
[8] D. Torrieri and K. Bakhru, "Frequency compensation in an adaptive antenna system for frequency-hopping communications," IEEE Trans. Aerosp. Electron. Syst., vol. AES-23, pp. 448-467, July 1987.

[9] R. E. Blahut, Digital Transmission of Information. Reading, MA: Addison-Wesley, 1990.

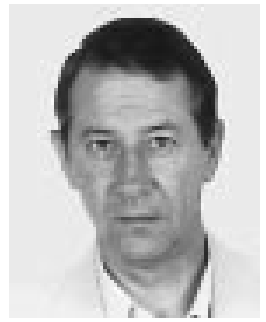

Miguel Angel Lagunas (F'97) was born in Madrid, Spain, in 1951. He received the Telecommunication Engineer degree from the Polytechnic University of Madrid (UPM) in 1973 and the Ph.D. telecom degree from the Polytechnic University of Catalunya (UPC), Barcelona, Barcelona, Spain.

From 1971 to 1973, he was a Research Assistant; from 1973 to 1979, he was a Teaching Assistant in network synthesis and semiconductor electronics; from 1979 to 1982, he was an Associate Professor of digital signal processing, and since 1983, he has been a Full Professor, teaching courses in signal processing, array processing, and digital communications at the Semiconductor Laboratory, ETSIT, Madrid. From 1981 to 1982, he received a Fulbright postdoctoral grant at the University of Colorado, Boulder. From 1987 to 1989, he was Project Leader of high speed SCMA and, from 1994 to 1995, ATM cable networks. He was Co-director of the first projects for the European Spatial Agency and the European Union, providing engineering demonstration models on smart antennas for satellite communications using DS and FH systems and mobile communications. From 1986 to 1989, he was Vice President for Research at UPC. He was Vice Secretary General for Research at CICYT from 1995 to 1996. His research activity is devoted to spectral estimation, adaptive systems, and array processing. His technical activities are in advanced front ends for digital communications combining spatial with frequency-time and coding diversity.

Dr. Lagunas was a Fellow Member in 1997 and a Member at Large in 1990 of Eurasip. He was elected Member of the Academy of Engineers of Spain in 1998.

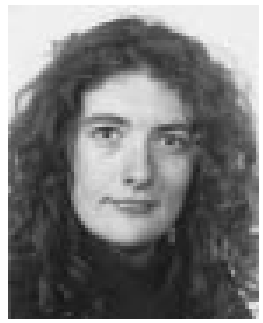

Ana I. Pérez Neira (S'92-M'95) was born in Zaragoza, Spain, in 1967. She graduated in telecommunication engineering in 1991 and received the Ph.D. degree in 1995 from the Universitat Politècnica de Catalunya (UPC), Barcelona, Spain.

In 1991, she joined the Department of Signal Theory and Communication of the UPC, where she carried on research activities in the field of higher order statistics and statistical array processing. She became an Assistant Professor in October 1992 and Associate Professor in December 1996. Her current teaching and research interests are in analog and digital signal processing, adaptive signal processing, and fuzzy processing, with applications to mobile/satellite communication systems.

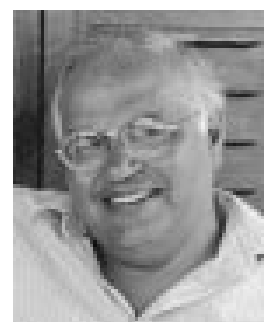

Moeness G. Amin (SM'91) received the B.Sc. degree in 1976 from Cairo University, Cairo, Egypt, the M.Sc. degree in 1980 from the University of Petroleum and Minerals, Dharan, Saudi Arabia, and the Ph.D. degree in 1984 from the University of Colorado, Boulder, all in electrical engineering.

In 1984, he joined The University of Colorado, Denver, as a Visiting Assistant Professor. He has been on the faculty of the Department of Electrical and Computer Engineering at Villanova University, Villanova, PA, since 1985, where he is now a Professor. His current research interests are in the areas of time-frequency analysis, spread spectrum communications, smart antennas, and blind signal processing.

Dr. Amin was an Associate Editor of the IEEE TRANSACTIONS ON SignaL PROCESSING and a member of the Technical Committee of the IEEE Signal Processing Society on Statistical Signal and Array Processing. He is currently a member of the IEEE Technical Committee on Signal Processing for Communications. He was General Chair of the 1994 IEEE International Symposium on Time-Frequency and Time-Scale Analysis and is a General Chair the IEEE Workshop on Statistical Signal and Array Processing to be held in the Pocono Mountains in Pennsylvania in August 2000. He was the recipient of the 1997 IEEE Philadelphia Section Award for "Outstanding Intellectual and Organizational Contributions to the IEEE Philadelphia Section in the Area of Signal Processing." He was also the recipient of the 1997 Villanova University Outstanding Faculty Research Award. Over the past four years, he has chaired three all-day workshops on Smart antennas, recent trends in adaptive filtering, and advanced signal processing applications in wireless communications. He is a member of Sigma Xi, Eta Kappa Nu, and Phi Kappa Phi.

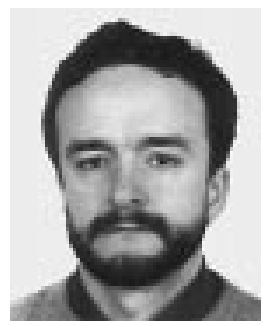

Josep Vidal (A'95) was born in 1965 in Barcelona, Spain. He received the telecommunication engineering and the Ph.D. degrees from the Polytechnic University of Catalonia (UPC), Barcelona, in 1989 to 1993 , respectively.

In 1988, he was with Cognivision Research, S.L., developing hardware for image analysis. From 1989 to 1990 , he was with the LSI at Ecole Polytechnique de Lausanne, Lausanne, Switzerland, as a Research Assistant working on digital signal processing for biomedical applications. In 1991, he was the recipient of the Ministry of Education grant to complete the Ph.D. thesis and joined the Signal Theory and Communications Department at UPC. In 1993, he became Lecturer, and since 1996, he has been an Associate Professor at UPC, where he teaches graduate and undergraduate courses in statistical signal processing, digital signal processors, mathematical methods for communications, and nonlinear signal processing. His current research interest is in statistical signal processing with application to mobile communication systems as well as DSP implementation aspects. He has published over 40 papers in international journals and conferences.

Dr. Vidal was co-organizer of the IEEE Signal Processing/ATHOS Workshop on Higher Order Statistics in Begur, Spain, in June 1995. 